\title{
ADMINISTRACIÓN ELECTRÓNICA: POR QUÉ IMPLANTAR UNA POLITICA DE GESTIÓN DE PROCESOS INSTITUCIONALES EN LAS ADMINISTRACIONES PÚBLICAS
}

\author{
e-Government: the need to implement a \\ business processes management policy in public \\ administration
}

María García-González

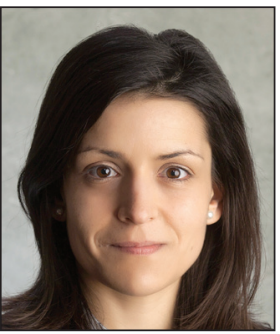

María García-González es documentalista especializada, e investigadora en el estudio, diseño e implementación de contenidos y herramientas para la óptima descripción de la gestión de datos, información y documentos tanto para la administración pública como para la empresa privada. Ha trabajado para el Congreso de los Diputados, el Senado, y el Ministerio de Defensa de España. Actualmente trabaja para la administración local. Licenciada y doctora en ciencias de la información y documentación, es profesora asociada en el Departamento de Información y Documentación de la Universidad de Murcia (UM). Forma parte del grupo de investigación Semiodoc UM. Sus temas de investigación son: calidad en los sistemas de gestión de contenidos en las organizaciones (ECM), procesos de negocio institucionales, gestión documental y archivística.

http://orcid.org/0000-0002-1640-9623

Universidad de Murcia, Facultad de Comunicación y Documentación Campus Universitario de Espinardo. 30100 Murcia, España mariasegunda.garcia@um.es

\section{Resumen}

Situación en que se encuentra la implantación de la administración electrónica en España y, concretamente, en las administraciones locales. Se ofrecen respuestas a un conjunto de cuestiones sobre cómo se está llevando a cabo ese proceso. Se observa que a pesar de las recomendaciones dadas a las administraciones públicas por normativas y directrices tanto europeas como nacionales, se están dejando en el camino varias fases definidas e identificadas que deberían solucionarse previamente. Nos referimos a los aspectos orgánico-funcionales antes que la búsqueda de soluciones técnicas y tecnológicas encaminadas a la implementación de metadatos.

\section{Palabras clave}

Administración electrónica; Gestión de procesos institucionales; Esquema de metadatos; Análisis funcional; Esquema nacional de interoperabilidad (ENI); Gestión de contenidos; Ciudadanos.

\section{Abstract}

The implementation of electronic government in Spain, specifically in local councils, is described. We tackle an array of questions about how this process is being carried out. We analyse whether recommendations given to the public sector by European and national rules and regulations are leaving behind several well-defined stages which should be previously solved. We are referring to the organic-functional aspects before looking for technical solutions and technical paths towards implementing metadata.

\section{Keywords}

e-Government; Business processes management; Metadata schema; Functional analysis; National scheme of interoperability (ENI); Contents management; Citizens.

García-González, María (2016). “Administración electrónica: por qué implantar una política de gestión de procesos institucionales en las administraciones públicas". El profesional de la información, v. 25, n. 3, pp. 473-483. 


\section{Implantación de la administración-e en España}

Los estudios sobre administración electrónica, y en particular los centrados en su desarrollo a nivel local, suelen plantearse desde una perspectiva exclusivamente nacional, sin profundizar en su tratamiento a nivel comunitario europeo a pesar de que el desarrollo de la administración electrónica se encuentra directamente condicionado por las decisiones adoptadas desde la Unión Europea (UE).

En España los estudios sobre administración electrónica local son insuficientes, y las corporaciones que se encargan directamente de ello suelen ser diputaciones provinciales, comunidades autónomas o la propia administración estatal, que suelen dar apoyo técnico a este nivel de la administración siguiendo normalmente directrices nacionales que a su vez siguen directrices europeas. Es sabido que no existe un modelo comunitario de administración electrónica completo y acabado, y menos aún de administración electrónica local.

No obstante, las actuaciones llevadas a cabo desde la UE vienen siendo cada vez más determinantes en la configuración de la administración electrónica. El eje vertebrador de la Ley 11/2007 sobre acceso electrónico de los ciudadanos a los servicios públicos (España, 2007) es el reconocimiento a los ciudadanos del derecho a comunicarse a través de medios electrónicos con las administraciones públicas y la consiguiente obligación de que éstas se doten de los medios y sistemas para que dicho derecho pueda ejercerse. Constituye una apuesta valiente y original formulada desde nuestro país con la que se cumple, en cierta medida, la obligación de habilitar el acceso por medios electrónicos a la información y a los procedimientos administrativos relacionados con la prestación de servicios que se exige desde el derecho comunitario, en concreto, a través de la Directiva 2006/123/CE (Unión Europea, 2006) relativa a los servicios en el mercado interior.

Los planes de modernización llevados a cabo por las administraciones públicas en los últimos años han ido encaminados a generar estructuras más eficientes para hacer efectiva la gestión de los procesos administrativos por medios electrónicos. En España, sin embargo, el marco de actuación en esta materia no acaba de ver la luz. Las administraciones locales, en su mayoría, son las organizaciones más perjudicadas ya que no han logrado adaptarse a las exigencias de esta nueva administración.

\section{Metodología}

El estudio de tres pilares fundamentales en los que se respalda el impulso de la administración electrónica, realizados en un trabajo inicial por García-González (2015), citados a continuación, y con la experiencia de más de ocho años trabajando en las administraciones públicas, fundamentan esa evidencia de la que se habla:

En primer lugar, el estudio de las directrices dadas desde la Unión Europea con la publicación de dos programas fundamentales que constituyen el Marco europeo de interoperabilidad-Europe interoperability framework (EIF)-:
- Interoperable delivery of European e-government services to public administrations, businesses and citizens (Idabc), (Unión Europea, 2004);

- programa para el período 2010-2015 denominado Interoperability solutions for European public administrations (ISA), (Unión Europea, 2009). Ambos, junto con la Ley 11/2007 de 22 de junio, de acceso electrónico de los ciudadanos a los servicios públicos (España, 2007) han permitido el desarrollo del Esquema nacional de interoperabilidad en España (ENI), quedando muy patente en el $R D$ 4/2010 de 8 de enero por el que se regula el Esquema nacional de interoperabilidad en el ámbito de la administración electrónica en España (España, 2010), que presenta un enfoque de acciones basado en elementos modulares, cuya finalidad es la creación de condiciones necesarias para garantizar el adecuado nivel de interoperabilidad técnica, semántica y organizativa en las administraciones públicas, de ahí la publicación de las diferentes normas técnicas de interoperabilidad (en adelante NTI).

En segundo lugar, la revisión de normativas y fuentes legales sobre administración electrónica tanto a nivel europeo como español, elaboradas desde el año 2000 con la Estrategia Lisboa 2000-2002 (Vida-Fernández, 2010), el Plan de acción 2010 (Comisión Europea, 2006), entre otros, y aquí en España la Ley paraguas (España, 2009a) y la Ley ómnibus (España, 2009b) propuestas a partir de los planes de acción europeos y la directiva comunitaria (Unión Europea, 2006), relativa a los servicios en el mercado interior, contribuyen a garantizar el acceso electrónico a los servicios públicos de una manera más eficiente, transparente y rentable. También, cabe destacar la publicación reciente de la Ley de procedimiento administrativo común de las administraciones públicas, PACA (España, 2015).

Los estudios en la implantación de la administración electrónica son escasos no sólo para las administraciones locales, sino también para las estatales y autonómicas

En tercer lugar, el estudio de las normas sobre calidad en la gestión de las organizaciones dedicadas a la gestión de documentos, a procesos de mejora continua en las organizaciones, y las dedicadas a la calidad en los sistemas de gestión de las organizaciones. Hablamos de todas aquellas normas UNE-ISO destinadas a ofrecer requerimientos para la mejora de los sistemas de gestión en las organizaciones 15489 (Aenor, 2006a; 2006b) 23081 (Aenor, 2008; 2011), 26122 (Aenor, 2008b), 9000 (Aenor, 2008c), 9001 (ISO, 2008), etc.

Por un lado, el análisis del conjunto de las fuentes abordadas en ese trabajo inicial nos permite ver cuál es el impacto aplicativo que están teniendo todas estas recomendaciones en la implantación de la administración electrónica, con respecto a la realidad vivida en las administraciones públicas. Por otro, el estudio de la metodología de trabajo que se plantea en el mismo trabajo de García-González (2015), 
a través del cual intenta solventar las carencias observadas en estas recomendaciones. Existe la necesidad de diseñar un modelo de descripción de procesos electrónicos, antes que implementar el uso de metadatos. De esta forma se obtendría un esquema conceptual de los procedimientos administrativos gestionados de forma electrónica, que posteriormente podrán traducirse a un lenguaje lógico. Todo ello nos conduce a proporcionar doce consideraciones necesarias para la puesta en funcionamiento de la administración electrónica:

\subsection{Abandonar la autonomía institucional en el cumpli- miento de objetivos generales para implantar la administra- ción electrónica}

En España el marco de actuación en materia de administración electrónica no acaba de ver la luz.

La Ley 11/2007 sobre acceso electrónico de los ciudadanos a los servicios públicos, es una apuesta ambiciosa que indica la obligación de habilitar el acceso por medios electrónicos a la información así como a los procedimientos administrativos relacionados con la prestación de servicios que se exige desde el derecho comunitario, en concreto, a través de la Directiva 2006/123/CE relativa a los servicios en el mercado interior y que, a partir del 31 de diciembre de 2009, todas las administraciones deberían haber puesto en marcha, medidas para que la e-administración fuese una realidad.

Existe una falta de compromiso, coordinación y colaboración en todos los niveles de la administración para implantar un verdadero Marco nacional de interoperabilidad

El hecho de que no exista un modelo comunitario de administración electrónica se debe a que las políticas sobre este tema se llevan a cabo a través de objetivos políticos generales, existiendo una autonomía institucional y procedimental en cada Estado miembro para la aplicación de esas políticas (Vida-Fernández, 2010). Se puede comprobar que los estudios sobre administración electrónica local son insuficientes. Muchas veces las actuaciones no se llevan a cabo hasta que no existe una necesidad real, haciéndolo de forma sectorial sin estudios previos o planificados.

Se sabe que el Marco europeo de interoperabilidad apuesta por la colaboración entre Estados miembros para conseguir unos objetivos comunes que permitan mejorar los servicios públicos para ciudadanos europeos por medios electrónicos y promover una puesta en práctica más eficiente de la políti- ca de la UE en esta materia, siendo perfectamente aplicable internamente a cada Estado miembro dentro de su estructura gubernativa (European Commission, 2011).

\subsection{Compromiso político en la implantación de la ad- ministración electrónica}

Las directrices europeas recomiendan que para establecer un Marco nacional de interoperabilidad debe existir un compromiso político, jurídico, organizativo y técnico de cada Estado miembro. En España ese compromiso jurídico se materializa en el conjunto de normas técnicas de interoperabilidad (NTI) publicadas. La legislación es la hoja de ruta para indicar a las administraciones públicas la forma de proceder, pero sin duda el funcionamiento de la administración electrónica queda mermado por cómo se está elaborando y aplicando realmente el conjunto de normas, y por la falta de definición del resto de compromisos que recomienda la Unión Europea por parte de nuestras administraciones públicas.

Las causas que pueden producir esta situación tienen mucho que ver con la falta de una estrategia de actuación bien diseñada sobre administración electrónica que implique a todos los niveles de nuestras administraciones.

A veces la división política hace que muchos de estos planes queden truncados o no lleguen a iniciarse. Todos los planes de actuación en esta materia requieren de una acción directa o indirectamente política, de una fuerte colaboración por parte de todas las escalas de la administración y de la asignación de recursos económicos. Creemos que para poner en funcionamiento un Marco nacional de interoperabilidad es necesario un verdadero compromiso político basado en criterios sólidos que se mantengan a lo largo del tiempo, aunque sabemos que las decisiones políticas están siempre condicionadas por la disponibilidad de recursos económicos. 
En este sentido las administraciones locales son las más perjudicadas, puesto que las decisiones políticas dependen principalmente de directrices nacionales y de la asignación o disponibilidad de recursos económicos.

\subsection{Se debe concebir la administración-e como un sistema de gestión integrado de información}

La administración electrónica no se debe entender única y exclusivamente como una única prestación de servicios al ciudadano a través de medios electrónicos, sino que debe concebirse como un sistema integrado de información donde todos los procesos de las administraciones públicas deben estar automatizados como una solución de gestión entre las mismas para la búsqueda y recuperación de información, permitiendo así la interoperabilidad en todos los niveles de la administración y por los mismos ciudadanos.

Por tanto, la administración electrónica es un sistema integrado de información virtual que funciona mediante el intercambio de información y la interacción entre los agentes.

En nuestras administraciones públicas existen varias fases definidas que deben solucionarse antes de poner en marcha la administración-e

\subsection{Solucionar previamente en todas las administra- ciones públicas los problemas orgánico-funcionales}

Las diferentes NTI pretenden cubrir las necesidades técnicas para ser aplicables en un planteamiento de partida basado en mínimos, de forma que se garantice la interoperabilidad entre las distintas administraciones, favoreciendo su implantación y aplicación en un corto período de tiempo con un impacto mínimo, pero sin perder una orientación de desarrollo y perfeccionamiento a lo largo del tiempo, en paralelo al progreso de los servicios de la administración electrónica, de las infraestructuras que los apoyan y de la evolución tecnológica (España, 2012c).

La capacidad que se necesita para afrontar un verdadero esquema nacional de interoperabilidad, por parte de las administraciones públicas, necesita solucionar previamente aspectos organizativos y semánticos (España, 2010).

Para que funcione la administración electrónica es necesaria una base normativa, metodológica-aplicativa y de asesoramiento. Es imprescindible la definición de una política de gestión integrada de información en las administraciones bien planificada de acuerdo con sus necesidades y posibilidades, y que integre una política de gestión de documentos electrónicos.

El nuevo modelo de administración que deseamos implantar precede a los requisitos tecnológicos que se han dado en las NTI. Solucionar los aspectos organizativos tiene que ver con la estructura orgánica y funcional de las instituciones, por lo que es necesario alinear todos los procesos y procedimientos efectuados en ellas para poder aplicar las NTI, en beneficio de esa administración electrónica.

\subsection{El análisis funcional como mejor método para la implantación de la administración electrónica}

Algunas de las teorías sobre el estudio de las funcionalidades institucionales plantean un análisis funcional de las organizaciones para evidenciar el contexto del documento. Proponen el análisis desde los estadios más altos del organigrama institucional para extraer esas funcionalidades. En contraposición se cree que, partiendo del análisis de lo más elemental, los documentos de un proceso institucional se pueden extraer mucho mejor las funcionalidades institucionales, es decir, obtener más datos de carácter colectivo o contextual.

Por tanto, las administraciones públicas deben apostar por un enfoque basado en procesos para definir su cuadro de clasificación funcional partiendo del análisis de los documentos. Es necesario documentar la gestión de los procesos de las administraciones públicas para un entorno electrónico que permitirá mejorar su tramitación, la recuperación de información, la prestación de servicios públicos al ciudadano y la interoperabilidad entre administraciones.

Se justifica esta posición debido a que los procesos quedan materializados en los expedientes, pero la gestión de los procesos no queda materializada en ellos. Los documentos pueden permitir documentar esa gestión, es decir, definir el proceso y desarrollar un modelo de descripción de procesos.

Entendemos que una organización gestiona una serie de procesos y cada unidad funcional -ya sean agentes, departamentos, servicios-implicada en uno de ellos, efectúa una serie de actividades sobre el mismo.

La gestión de un proceso se refiere a las actividades desempeñadas para su tramitación y que se pueden llamar "procedimientos", incluyendo aquellos destinados a la gestión del documento -acciones efectuadas sobre el propio documento-. Documentar esta gestión conduce a la obtención de una estructura conceptual del proceso real evaluado que será traducida a una estructura lógica para la administración-e mediante el uso de metadatos (García-González, 2015).

\section{El estudio del documento administrativo y su contexto son los elementos clave para definir los procesos de la adminis- tración-e}

El documento no es sólo el único protagonista para evidenciar la gestión de los procesos de las administraciones públicas. El análisis de las regulaciones que afectan a un proceso y la evaluación de la práctica real llevada a cabo en la tramitación del mismo también garantizan el poder documentar su gestión.

\subsection{Solucionar las tareas pendientes en materia de gestión orgánico-funcional antes de implantar la ad- ministración electrónica}

El 26 de julio de 2012 se publicó en España la norma técnica de interoperabilidad de "Política de gestión de documentos electrónicos" y en noviembre de 2013 el "Modelo de política 
de gestión de documentos electrónicos" (Dirección General de Modernización Administrativa, Procedimientos e Impulso de la Administración Electrónica, 2012). Con la publicación de estas normativas, por primera vez empieza a ser efectivo el Marco nacional de interoperabilidad. Como puede apreciarse, se trata de normas muy jóvenes que demandan a las administraciones actuaciones, indicando "qué" deben y en cierta medida "cómo" deben hacerlo para elaborar una verdadera política de gestión de documentos electrónicos de acuerdo con el Esquema nacional de interoperabilidad.

Analizando las directrices marcadas por la NTI de "política de gestión de documentos electrónicos" se aprecian varias fases definidas que deben solucionarse por parte de las administraciones antes de llegar a la implementación de metadatos, donde muchas de ellas ni siquiera han superado la primera fase -nos referimos a las administraciones localesy que definimos a continuación:

- La condición de obligatoriedad en el desarrollo de una verdadera política de gestión de documentos electrónicos por parte de las administraciones públicas de nuestro país.

- Observar la capacidad de autosuficiencia de cada uno de los niveles de nuestras administraciones públicas, pues no todas pueden asumir estas competencias. La falta de recursos materiales y humanos, así como la falta de conocimiento especializado y cualificado en procesos de gestión de documentos electrónicos y cuanto menos en la implementación de metadatos, hacen que este cometido sea una tarea casi imposible, sobre todo para las administraciones locales.

- Muchas de ellas deben solucionar todavía problemas de gestión, es decir, de tratamiento y descripción documental y, como es de esperar, de implantación de un sistema de gestión documental (SGD) adaptado a las necesidades de gestión de las propias administraciones, pues la gestión documental depende en parte de una cuestión económica y solidaria por parte de otras administraciones, tales como diputaciones provinciales $\mathrm{o}$ autonómicas, al menos en las administraciones locales de pequeña-mediana población.

- Estas administraciones deben afrontar el problema acerca de la dualidad encontrada, por un lado, de administración en papel y, por otro, de administración-e que, a veces, responden a una serie de actuaciones aisladas-soluciones de gestión- por parte de terceros y que carecen de una base sólida para mantenerse por las siguientes circunstancias: planificación en la formación del personal, cuestiones políticas por ambas administraciones, cuestiones económicas, falta de recursos humanos y técnicos. Las administraciones locales deberán tomar medidas para solventar internamente esta situación y gestionar de alguna manera la ayuda proporcionada por terceros con el fin de sacarle la máxima rentabilidad a los trabajos prestados para definir una política propia de gestión de documentos electrónicos, que permita así establecer unos criterios internos sólidos de actuación. Esta carencia repercute, por ejemplo, en que existen procedimientos que se efectúan de forma electrónica y otros de forma física en papel, sin tener un control sobre los primeros. Se necesitan, por tanto, soluciones que contemplen estos problemas.

- Disponer de una plantilla de profesionales -documentalistas, informáticos, etc.-, que participen en la definición de las políticas y efectúen las tareas de gestión integral de la información institucional, incluida la gestión documental, seleccionando o diseñando las herramientas tecnológicas adecuadas a las necesidades de gestión.

- Disponer de expertos que conozcan la estructura organizativa y funcional de las administraciones locales para poder diseñar una arquitectura de procesos bien definida y que, en colaboración con los profesionales anteriores, diseñen modelos de datos "activos semánticos" que permitan describirlos tal y como establece la NTI de Relación de modelo de datos y el RD 4/2010 ENI para su posterior publicación en el Centro de Interoperabilidad Semántica (CISE) del Ministerio de Industria, Energía y Turismo, como una tarea colaborativa de optimización de procesos y trabajo compartido para conseguir el intercambio de información entre administraciones.

Las intenciones de la NTI de Política de gestión de documentos electrónicos y el Modelo de política de gestión de documentos electrónicos son buenas, pero muchas de las ad-

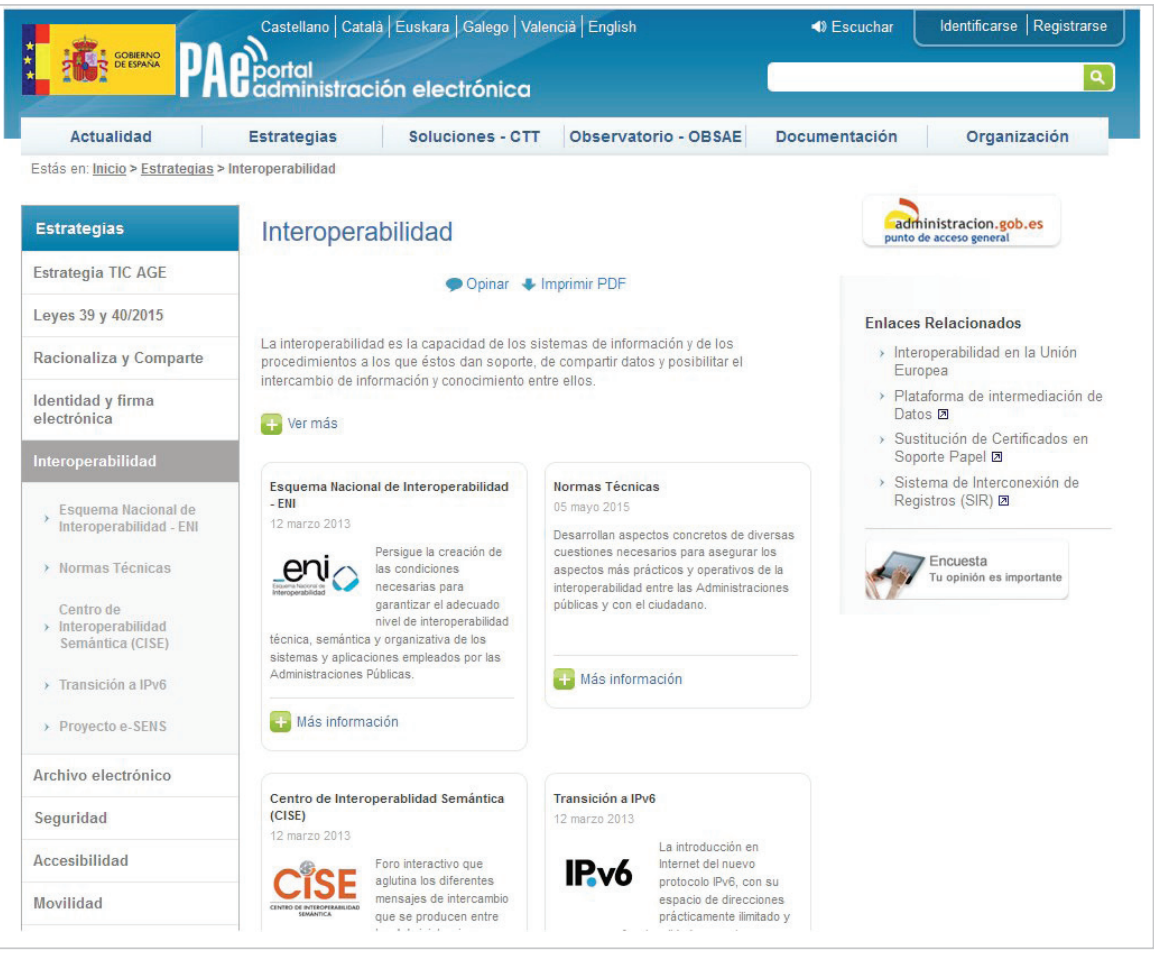

http://administracionelectronica.gob.es/pae_Home/pae_Estrategias/pae_Interoperabilidad_Inicio.html 
ministraciones locales de nuestro país no pueden tomarlas como referencia porque tienen pendientes otras tareas previas en las que ocuparse. Convendría categorizar cada una de las administraciones en función de sus capacidades y posibilidades, adaptando las NTI y acotando distancias entre el estado tan avanzado de desarrollo de las administraciones nacionales y autonómicas en materia de gestión documental, en comparación con las administraciones locales.

\subsection{Ir hacia un nuevo marco legislativo sobre dere- cho administrativo electrónico}

Según Barnés-Vázquez (2010), el procedimiento administrativo se agota en lo que la Ley 30/1992 (España, 1992) o en su versión electrónica la Ley 11/2007 (España, 2007) hayan establecido, cuando esta última no es más que la versión digital de esa primera generación de procedimientos, dejando fuera muchas otras cosas. Se trata más de una ley de medidas que de una norma con un esfuerzo de adaptación al nuevo medio.

Los textos normativos actuales sobre procedimiento administrativo son insuficientes para regular los nuevos procedimientos administrativos electrónicos

El nuevo modelo de administración demanda nuevos procedimientos que la legislación en materia de derecho administrativo no contempla. Se trata de procedimientos que van mucho más allá de la función adjetiva de la que nos habla Barnés -basada en un carácter instrumental al servicio del derecho material- puesto que su objetivo es hallar soluciones que no han sido previstas en las leyes y que deben regularse. Esta tarea deberá encomendarse al legislador, a condición de que observe un sofisticado procedimiento deliberativo y de búsqueda de consenso, de colaboración y participación a todos los niveles de la administración.

En el caso de España, las políticas públicas adoptadas y materializadas en las normas técnicas de interoperabilidad demandan nuevos procedimientos - denominados de "tercera generación" según Barnés-. Por tanto, se deben adoptar formas de gobierno y de regulación que reflejen las nuevas prácticas de trabajo de las administraciones $y$, posteriormente, hacer que las tecnologías de la información respondan a sus requerimientos, potenciando el nuevo concepto de administración cooperativa o de interacción.

Parece que la Ley 39/2015, es consciente y demuestra que es necesario ponerse a ello, indicando lo siguiente:

“[...] la tramitación electrónica no puede ser todavía una forma especial de gestión de los procedimientos sino que debe constituir la actuación habitual de las administraciones. Porque una administración sin papel basada en un funcionamiento íntegramente electrónico no sólo sirve mejor a los principios de eficacia y eficiencia, al ahorrar costes a ciudadanos y empresas, sino que también refuerza las garantías de los interesados. En efecto, la constancia de documentos y actuaciones en un archivo electrónico facilita el cumplimiento de las obligacio- nes de transparencia, pues permite ofrecer información puntual, ágil y actualizada a los interesados [...].

[...] resulta clave contar con una nueva ley que sistematice toda la regulación relativa al procedimiento administrativo, que clarifique e integre el contenido de las citadas Ley 30/1992, de 26 de noviembre y Ley 11/2007, de 22 de junio, y profundice en la agilización de los procedimientos con un pleno funcionamiento electrónico. Todo ello revertirá en un mejor cumplimiento de los principios constitucionales de eficacia y seguridad jurídica que deben regir la actuación de las administraciones públicas". (España, 2015).

Es necesario que cada administración defina los procedimientos de cada proceso ejecutados internamente. Será preciso un esfuerzo concertado para establecer un método de trabajo que permita armonizar y categorizar los procesos para todos los niveles de la administración.

\subsection{Implantar un sistema que permita describir las funciones de la administración-e}

Con la aplicación de un "modelo de descripción de procesos" se demuestra, en primer lugar, que sólo y cuando se solucionen en nuestras administraciones públicas los aspectos orgánico-funcionales para la administración-e se podrán solucionar los aspectos técnico-semánticos. Documentar la gestión de un proceso real sirve de indicio para solucionar el problema inicial planteado, ofreciendo así resultados válidos para afrontar una segunda etapa de actuación.

La evaluación y análisis de posibles resultados tras la aplicación de un "modelo de descripción de procesos" pueden plantear dos interrogantes que deben tenerse en cuenta para la posterior aplicación de un modelo de metadatos: ¿hasta qué punto es necesario describir la gestión de un proceso real para la administración-e? y ¿cómo se debe documentar la gestión de un proceso? (García-González, 2015).

\subsection{Ventajas en la normalización del procedimiento administrativo para la administración electrónica}

Las regulaciones no nos dicen todo acerca de los procesos ejecutados en las administraciones públicas: sólo permiten interpretar la tramitación de los procesos pero no indican "quién", "qué" y "cómo" hace o se hace para cada proceso. Se pueden deducir algunos de los Procedimientos de tramitación normal del proceso pero no todos. Como resultado final, la materialización de los procedimientos en los documentos tampoco dicen "qué", "cómo" y "cuántos", y la secuencia de los procedimientos de gestión del documento se efectúan en su ejecución para una administración-e.

Existen motivos suficientes para plantear la normalización de funciones de un proceso dentro de las administraciones públicas para un entorno electrónico. Se trata de una tarea bastante complicada que requiere (García-González, 2015):

- Determinar el alcance del proceso.

- Unificar criterios por características similares de los procesos.

- Categorizarlos por cada administración.

- Extraer todos los componentes de un proceso. 
- Determinar hasta qué punto se puede aplicar la traducción de lo indicado en el procedimiento administrativo común y en las regulaciones que afectan al proceso para un entorno electrónico. Se puede decir que son muchas las regulaciones de carácter general que afectan a la actividad de una organización, existiendo muy pocas de carácter específico que regulen los componentes de un proceso para su interpretación, y como es de esperar tampoco a procesos electrónicos.

- Delimitar cómo y hasta dónde se deben traducir las funciones de procesos reales producidos en las administraciones públicas a funciones de procesos electrónicos producidos para la administración-e.

- Definir y regular/normalizar los Procedimientos de tramitación normal (PTN) y Procedimientos de gestión del documento (PGD) de los procesos.

- Identificar y definir procesos paralelos o subprocesos.

- Numerar las secuencias de procedimientos en el tiempo. Es importante reflejar los tiempos límite normalizados para efectuar una acción o gestionar y tener preparado un documento/s, o ambos casos. Esta información será de utilidad para la adecuada gestión del proceso en un entorno electrónico.

- Definir los tipos de agentes implicados en el proceso -de acuerdo a la estructura orgánico-funcional y del tamaño de las instituciones-.

- Asignación de roles y competencias para la ejecución de los procedimientos del proceso.

- Representar y definir las relaciones entre agentes implicados en la tramitación del proceso, con la acción que efectúan dentro del proceso y con los elementos que producen o gestionan-documentos/expedientes-. El hecho de plasmar las relaciones entre entidades internas y, sobre todo, las externas de un proceso, demuestra que efectivamente la interoperabilidad entre administraciones es posible y debe existir la colaboración de trabajo común para llevar a cabo los objetivos de la administración-e porque así lo exige el proceso -garantizando la rapidez, eficacia, eficiencia de nuestras administraciones públicas en la prestación de servicios-, donde dos o tres administraciones públicas interaccionan entre sí, incluso con los ciudadanos ejecutando los procedimientos de un proceso.

- Establecer los tipos de vínculos entre elementos de un proceso.

Todas estas actuaciones garantizan el poder presentar la estructura conceptual que permitirá diseñar una estructura lógico-semántica del proceso mediante metadatos.

\subsection{Antes de la implementación de metadatos es imprescindible obtener un esquema conceptual del procedimiento administrativo para que sea gestiona- do por medios electrónicos}

Desde el punto de vista semántico se observa que son muchas las corrientes teóricas que desde los años 90 recomiendan el uso de metadatos para la descripción de recursos electrónicos. Todavía hoy queda mucho por recorrer debido a que los metadatos están tomando nuevos usos para describir no sólo documentos, sino también para describir aquellos procesos que los generan: sus elementos componentes y los flujos de secuencias en ellos producidos.
La descripción de procesos mediante un modelo entidadrelación permite obtener una estructura lógica normalizada para un entorno virtual. Las posibilidades que ofrece permiten determinar el grado de descripción del proceso mediante la asignación de metadatos. A partir de esta consideración, la administración-e debe concebirse como un verdadero sistema integrado de información dispuesto en estructuras lógicas normalizadas. Por tanto, y de acuerdo con los estándares de metadatos, es cierto que analizando cualquier actividad de una institución las principales entidades de metadatos que se pueden establecer para la descripción de un proceso son: Documento, Agente, Actividad, Regulación y Relación entre entidades.

Las NTI ofrecen planteamientos de partida basados en mínimos que no son de obligado cumplimiento para las administraciones públicas, cuando éstas deberían haber superado ya el compromiso que tenían de poner medios para que la e-administración fuera una realidad a partir del 31 de diciembre de 2009

Son muchas las posibilidades que nos ofrecen estos modelos: por tanto, para el proceso que se seleccione de cualquier administración pública es muy importante describir todos los elementos que lo componen.

La posibilidad de estructuración y representar los procesos en una arquitectura lógica mediante metadatos garantiza (García-González, 2015):

- Representar de forma homogénea la información.

- Registrar para cada componente del proceso la información necesaria para su gestión y las relaciones existentes en él, que enlazan los agentes con sus acciones, estas acciones con otras, así como con los documentos y expedientes.

- Garantizar que para cada acción efectuada en el proceso se registre información sobre el momento de su ejecución, el tipo de acción, los agentes y los permisos de acceso para llevarla a cabo en un entorno electrónico.

- Asegurar que se registre para los documentos y expedientes la información de contenido y contexto (agente gestor, vida útil, accesibilidad, seguridad, períodos de gestión, conservación).

- Registrar y vincular información sobre las regulaciones que se le aplican al proceso.

- Facilitar la recuperación de información sobre el proceso mediante la utilización de lenguajes controlados y ayudar a diseñar ecuaciones de búsqueda precisas que proporcionen información relevante.

- Permitir contextualizar de forma dinámica las necesidades de información y gestión de los usuarios.

- Mejorar la comunicación entre agentes.

- Intercambiar información entre administraciones, de manera uniforme y normalizada.

- Establecer y normalizar los permisos de accesibilidad de 
los agentes para gestionar el proceso.

- Controlar, modificar, eliminar y conservar la información del proceso.

- Normalizar la gestión de un proceso electrónico.

- Describir y gestionar documentos o expedientes sin la necesidad de implantar un sistema de gestión de documentos.

- Registrar la información de los vínculos producidos entre estructuras lógicas.

- Compatibilidad: ayudar a planificar la migración de datos y otras necesidades de conservación.

- Proporcionar una referencia para evaluar la calidad de la gestión de procesos dentro y entre organizaciones, a efectos de auditoría y control.

- Ampliar o extender semánticamente los niveles de descripción de acuerdo con las necesidades de información.

- Servir de base para generar descripciones enriquecidas de nuevos servicios electrónicos.

\subsection{Convivencia: administración en papel y/o admi- nistración electrónica}

Dentro del conjunto de normas técnicas publicadas bajo el marco del Esquema nacional de interoperabilidad (ENI) se observa cierta ambigüedad al analizar estos tres cuerpos documentales: el propio $R D$ 4/2010 ENI que lo regula, la NTI sobre política de gestión de documentos electrónicos y la documentación complementaria en la que se desarrolla el esquema de metadatos propuesto para la gestión de documentos electrónicos en nuestras administraciones públicas (Dirección General de Modernización Administrativa, Procedimientos e Impulso de la Administración Electrónica, 2012).

El RD 4/2010 ENI define metadatos de gestión de documentos como:

"información estructurada o semiestructurada que hace posible la creación, gestión y uso de documentos a lo largo del tiempo en el contexto de su creación. Sirven para identificar, autenticar y contextualizar documentos, y del mismo modo a las personas, los procesos y los sistemas que los crean, gestionan, mantienen y utilizan".

El ENI habla de metadatos de creación, gestión y uso, pero no de preservación, cuando verdaderamente dentro del conjunto de normas técnicas del ENI se encuentra que el modelo español de metadatos diseñado atiende a aspectos relacionados con la preservación del documento físico y electrónico.

El modelo español de metadatos de gestión de documentos electrónicos, el modelo australiano y la norma UNE-ISO 23081, también indican que los sistemas diseñados para gestionar los documentos necesitan metadatos como base para los procesos de gestión de documentos, y que uno de los principales usos de los metadatos es la representación de entidades del entorno del documento en el sistema de gestión. De ahí que los metadatos se puedan aplicar a diferentes niveles de agregación, documentos individuales, conjuntos o agregaciones de documentos, o sistemas completos de gestión de documentos -expedientes, series, fondos-. Todos ellos como entidades que rodean al documento.
Se cree que lo correcto y efectivo para la administración-e es utilizar metadatos para describir a nivel de ítem o documento -no a nivel de serie o fondo-. La preocupación debe ser por la preservación del documento electrónico donde lo válido es la recuperación de información por contenidos.

A la hora de poner en funcionamiento la administración-e, de acuerdo con las directrices que establecen las normas para que cada administración desarrolle su propia política de gestión de documentos electrónicos e implante el modelo español de metadatos propuesto, sería necesario solventar estas ambigüedades.

La importancia de la práctica archivística en el entorno digital repercute en la implantación de programas para la gestión de documentos de archivo electrónicos. En la actualidad la gestión documental se encuentra orientada a aspectos relacionados con los procesos que crean y manejan los documentos de archivo y los sistemas donde se mantienen, por lo que las administraciones públicas van encaminadas hacia la implantación de programas o aplicaciones de gestión de procesos que integren la gestión de documentos.

\section{El ciclo de vida del documento llevado a cabo en la práctica archivística debe quedar reflejado en los procesos que se diseñen para la administración-e}

Actualmente, para la gestión documental, tiene mayor relevancia el control que se pueda ejercer sobre los documentos de archivo a partir de su creación y hasta su disposición final como por ejemplo si deben ser eliminados o transferidos a un archivo histórico-. Identificar estos procedimientos garantizará o no su preservación a largo plazo, su autenticidad, integridad, fiabilidad, control y acceso en un entorno electrónico.

Hasta ahora, las buenas prácticas para los documentos de archivo tenían como base los sistemas de gestión documental; actualmente todos estos procedimientos deberán quedar documentados en los nuevos sistemas de gestión de procesos para la administración-e y, finalmente, ser descritos mediante metadatos.

En conclusión, las administraciones públicas deben tomar una decisión para adaptar la antigua práctica archivística de gestión documental a la nueva realidad de gestión de procesos tramitados electrónicamente, los cuales tienen como resultado la producción de los documentos y la materialización en expedientes electrónicos. Deben determinar si utilizar metadatos de preservación para los documentos electrónicos y si los nuevos sistemas de gestión también se encargarán de describir aspectos físicos del documento en papel.

\subsection{La administración-e en la web 3.0}

La web 2.0 presenta nuevas alternativas de estructuración de la información web o de los contenidos que se encuentran en la Red. La nueva forma de trabajo en las organizaciones, ya sean empresas privadas o administraciones públicas, va encaminada a una gestión integrada de información o de contenidos que demanda un flujo de comunicación constante de información, cuyo soporte es la Red y que se espera que dará lugar a una red inteligente: la web semántica. 
Poco a poco vemos que esas estructuras de información se están normalizando con el uso de metadatos. Sabemos que los metadatos, en el ámbito de internet, se pueden definir como todos aquellos datos comprensibles por el ordenador estructurados de tal forma que son utilizados para localizar, identificar y describir el contenido de un espacio web y, por lo tanto, para la búsqueda y recuperación de información (Chain-Navarro; Mas-Bleda, 2007).

Se cree que la descripción de acciones o funciones ejecutadas para la tramitación de los procesos administrativos en la administración electrónica, mediante el uso de metadatos, desembocará en la normalización de esas estructuras lógicas para la nueva web 3.0.

\section{Conclusiones}

El conjunto de normas y directrices publicadas para el impulso de la administración-e supone una apuesta ambiciosa que ofrece una guía sobre la ruta que deben adoptar todas las administraciones públicas en nuestro país. Creemos, y de hecho es evidente, que no todos los niveles de la administración pública española pueden cumplirlas, incluso aquellas que disponen de más recursos como pueden ser las administraciones estatales.

Sabemos que las directrices no son de obligado cumplimiento, pero todas las administraciones a partir del 31 de diciembre de 2009 debieron haber puesto medidas para que la e-administración fuese una realidad. A día de hoy nos encontramos con ciertos objetivos no cumplidos de los propuestos por la Ley 11/2007 sobre acceso electrónico de los ciudadanos a los servicios públicos (España, 2007). La insuficiente gestión de trámites y la falta de una buena planificación para el desarrollo de una estrategia adecuada en nuestras administraciones, hace que las expectativas de la ley no se hayan cumplido.

Para las administraciones locales supone un verdadero esfuerzo el cumplimiento de las normas técnicas de interoperabilidad publicadas bajo el amparo del $R D 4 / 2010$ por el que se regula el Esquema nacional de interoperabilidad, desarrollado a partir de esa primera ley (Ley 11/2007) en el ámbito de la administración-e. Tan sólo analizando estos cuatro cuerpos documentales de normas, citados a continuación, publicados en la web de la Dirección General de Modernización Administrativa, Procedimientos e Impulso de la Administración Electrónica, podemos justificar el porqué:

a) El primero, la NTI sobre Relación de modelos de datos (España, 2012b) fundamentada en la disposición adicional primera del $R D 4 / 2010$ de 8 de enero por el que se regula el Esquema nacional de interoperabilidad, en la que se indica que cada administración debe poner a disposición del Centro de Interoperabilidad Semántica (CISE) los modelos de datos para compartir información. Este centro persigue la recopilación de todos los modelos de datos diseñados en cada una de las administraciones públicas para facilitar la reutilización de los mismos y, en una labor posterior, proceder a la identificación de modelos de datos comunes.

b) El segundo, NTI sobre Política de gestión de documentos electrónicos (España, 2012a), en la que se hace una valoración sobre la importancia de la utilización de metadatos de acuerdo con los términos establecidos en el $R D 4 / 2010$, en la cual se definen los metadatos de gestión de documentos.

c) El tercero, el documento que presenta el Esquema de metadatos para la gestión del documento electrónicos (e-Emgde) (Dirección General de Modernización Administrativa, Procedimientos e Impulso de la Administración Electrónica, 2012), el cual puede ser utilizado como apoyo para la adecuación de cada organización a los requisitos de interoperabilidad en materia de gestión de documentos.

d) Finalmente, en cuarto lugar, el Modelo de política de gestión de documentos electrónicos (Dirección General de Modernización Administrativa, Procedimientos e Impulso de la Administración Electrónica, 2012), en el que se pone de manifiesto, por primera vez, el marco de normas ISO establecidas, relacionadas con la necesidad de documentar la gestión del documento electrónico. Es aquí donde se atisba por primera vez el comprometerse en la labor de documentar el contexto del documento (es decir documentar las acciones que son la causa de su materialización).

Observando los resultados obtenidos en el trabajo de investigación de García-González (2015), el cumplimiento de las directrices que dictan estas normas en el tiempo deberían hacerse a la inversa. En primer lugar, todas las administraciones deberían haber adoptado y saber ya cómo elaborar una política de gestión de documentos electrónicos, tal y como presenta el Modelo de política de gestión de documentos electrónicos y la reciente Ley 39/2015 del procedimiento administrativo común de las administraciones públicas (España, 2015) de sistematización de toda la regulación del procedimiento administrativo (dividido el bloque de legalidad en dos leyes, la denominada Ley de régimen jurídico del sector público, y por otro lado, la Ley del procedimiento administrativo común de las administraciones públicas. Ocupándose la primera ley de la administración mirándose el ombligo, ad intra: personas, órganos y funcionamiento, así como relaciones; la segunda ley se ocupa de la administración mirando hacia el ciudadano, ad extra: procedimientos de relación entre administración y ciudadano) (Chavés, 2015). Entendiendo que primero debe solucionarse ese carácter orgánico-funcional de las administraciones para posteriormente integrarse y cumplir con las directrices de las NTI de Relación de modelo de datos (uso y publicación de metadatos).

No se puede pedir a las administraciones locales que publiquen sus "activos semánticos", es decir, comiencen a implementar metadatos cuando todavía se aprecian varias fases definidas que deben solucionarse, como, por ejemplo, el diseñar una política de gestión de documentos electrónicos que finalmente quede integrada dentro de una política de gestión de procesos institucionales para nuestras administraciones públicas.

La realidad es que, si analizamos las últimas publicaciones de normas y directrices que ofrece la Dirección General de Modernización Administrativa, Procedimientos e Impulso de la Administración Electrónica, así como el conjunto de normas ISO, y las contrastamos con la práctica real que se ejerce en las administraciones locales, éstas conducen a la adopción de un enfoque por procesos institucionales, estando ya en la fase de imponer una política de gestión de pro- 
cesos electrónicos cuando todavía, en muchas de nuestras administraciones, sobre todo las locales, aún no se tiene diseñada una política de gestión documentos electrónicos, estando todavía en la fase de sistematización como indica la actual Ley 39/2015 (García-González, 2015). Solucionarlo debería ser el objetivo primordial para que nuestras administraciones públicas tengan definitivamente operativa esa administración-e.

Con la publicación de la Ley 39/2015 el Estado es consciente de que siempre habrá administraciones institucionales, locales e incluso alguna autonómica que no sepa, no quiera o no pueda adaptarse a las exigencias electrónicas en tiempo, por lo que el Estado se ofrece a ayudar y suplir, pero eso sí, cobrando (Chavés, 2015).

Por eso establece la disposición adicional 2a:

"Para cumplir con lo previsto en materia de registro electrónico de apoderamientos, registro electrónico, archivo electrónico único, plataforma de intermediación de datos y punto de acceso general electrónico de la administración, las comunidades autónomas y las entidades locales podrán adherirse voluntariamente y a través de medios electrónicos a las plataformas y registros establecidos al efecto por la Administración General del Estado. Su no adhesión, deberá justificarse en términos de eficiencia conforme al artículo 7 de la Ley orgánica 2/2012, de 27 de abril, de estabilidad presupuestaria y sostenibilidad financiera. Asimismo, la citada adhesión podrá conllevar la repercusión de los costes económicos que se generen" (España, 2015).

Hay que tener en cuenta que la vigencia de la ley se contempló a un año aunque con las correspondientes adaptaciones se prolongará. Esperemos que las expectativas de la nueva ley arrojen un marco de trabajo que permita gozar de autonomía a aquellas administraciones como pueden ser las locales para poder equipararse, en esta implementación, al resto de niveles de nuestras administraciones públicas como pueden ser las estatales o autonómicas, alcanzando la efectiva operatividad e interoperabilidad entre ellas a través de la administración-e en conjunto, y con los ciudadanos.

\section{Bibliografía}

Aenor (2006a). Información y documentación. Gestión de documentos. Parte 1: Generalidades. UNE-ISO 154891:2006. Madrid: Aenor.

Aenor (2006b). Información y documentación. Gestión de documentos. Parte 2: Directrices. UNE-ISO/TR 154892:2006. Madrid: Aenor.

Aenor (2008a). Información y documentación. Procesos de gestión de documentos. Metadatos para la gestión de documentos. Parte 1: Principios. UNE-ISO 23081-1:2008. Madrid: Aenor.

Aenor (2008b). Información y documentación. Análisis de los procesos de trabajo para la gestión de documentos. UNE-ISO/TR 26122:2008 IN. Madrid: Aenor.

Aenor (2008c). Sistemas de gestión de la calidad. Requisitos. UNE-EN ISO 9001:2008. Madrid: Aenor.

Aenor (2011). Información y documentación. Procesos de gestión de documentos. Metadatos para la gestión de do- cumentos. Parte 2: Elementos de implementación y conceptuales. UNE-ISO 23081-2:2008. Madrid: Aenor.

Barnés-Vázquez, Javier (2010). "El procedimiento administrativo y el gobierno electrónico". Claves del gobierno local, n. 12 , pp. 77-100.

http://repositorio.gobiernolocal.es/xmlui/handle/10873/968

Chain-Navarro, Celia; Mas-Bleda, Amalia (2007). "Metacontenidos en los sistemas webs de los principales ayuntamientos españoles". Tejuelo, n. 7, pp. 29-44.

http://www.anabadmurcia.org/ojs/index.php/tejuelo/article/ view/39

Chavés, José (2015). “El BOE alumbra siamesas administrativas: Ley 39/2015 de procedimiento y Ley 40/2015 de régimen jurídico". DelaJusticia.com, 2 octubre.

http://contencioso.es/2015/10/02/el-boe-alumbra-siamesasadministrativas-ley-392015-de-procedimiento-y-ley-402015de-regimen-juridico

Comisión Europea (2006). Comunicación de la Comisión al Consejo, al Parlamento Europeo, al Comité Económico y Social Europeo y al Comité de las Regiones. Plan de acción sobre administración electrónica i2010. Acelerar la administración electrónica en Europa en beneficio de todos.

http://eurlex.europa.eu/legal-content/ES/TXT/?uri=celex\%3A52006DC0173

Dirección General de Modernización Administrativa, Procedimientos e Impulso de la Administración Electrónica (2012). Política de gestión de documentos electrónicos.

http://administracionelectronica.gob.es/pae_Home/pae_ Estrategias/pae_Interoperabilidad_Inicio/pae_Normas_ tecnicas_de_interoperabilidad.html

España (1992). "Ley 30/1992, de 26 de noviembre, de régimen jurídico de las administraciones públicas y del procedimiento administrativo común". BOE, n. 285, 27 de noviembre, pp. 40300-40319.

http://www.boe.es/buscar/doc.php?id=BOE-A-1992-26318

España (2007). “Ley 11/2007, de 22 de junio, de acceso electrónico de los ciudadanos a los servicios públicos". BOE, n. 150, 23 de junio, pp. 27150-27166.

http://www.boe.es/diario_boe/txt.php?id=BOE-A-2007-12352

España (2009a). "Ley 17/2009, de 23 de noviembre, sobre el libre acceso a las actividades de servicios y su ejercicio". $B O E$, n. 283, 24 de noviembre, pp. 99570-99593.

http://www.boe.es/buscar/doc.php?id=BOE-A-2009-18731

España (2009b). "Ley 25/2009, de 22 de diciembre, de modificación de diversas leyes para su adaptación a la ley sobre el libre acceso a las actividades de servicios y su ejercicio". $B O E$, n. 308, 23 de diciembre, pp. 108507-108578. http://www.boe.es/buscar/doc.php?id=BOE-A-2009-20725

España (2010). "Real decreto 4/2010, de 8 de enero, por el que se regula el Esquema nacional de interoperabilidad en el ámbito de la administración electrónica". BOE, n. 25, 29 de enero, pp. 8139-8156.

http://www.boe.es/buscar/doc.php?id=BOE-A-2010-1331

España (2012a). "Resolución de 28 de junio de 2012, de la Secretaría de Estado de Administraciones Públicas, por la que se aprueba la Norma técnica de interoperabilidad de política de gestión de documentos electrónicos". BOE, n. 
178, 26 de julio, pp. 53776-53780.

https://www.boe.es/diario_boe/txt.php?id=BOE-A-2012-10048

España (2012b). "Resolución de 28 de junio de 2012, de la Secretaría de Estado de Administraciones Públicas, por la que se aprueba la Norma técnica de interoperabilidad de relación de modelos de datos". BOE, n. 178, 28 julio, pp. 53793-57807. http://www.boe.es/diario_boe/txt.php?id=BOE-A-2012-10050

España (2012c). "Resolución de 3 de octubre de 2012, de la Secretaría de Estado de Administraciones Públicas, por la que se aprueba la Norma técnica de interoperabilidad de catálogo de estándares". BOE, n. 262, 31 de octubre, pp. 76713-76723. https://www.boe.es/diario_boe/txt.php?id=BOE-A-2012-13501

España (2015). “Ley 39/2015, de 1 de octubre, del procedimiento administrativo común de las administraciones públicas". BOE, n. 236, de 2 de octubre, pp. 89343-89410. https://www.boe.es/diario_boe/txt.php?id=BOE-A-2015-10565

García-González, María (2015). Administración electrónica: de la evidencia de la gestión de documentos a la documentación de la gestión de procesos institucionales locales. Tesis doctoral. Murcia: Universidad de Murcia, Facultad de Comunicación y Documentación.

https://digitum.um.es/xmlui/handle/10201/45706

ISO (2008). Conjunto de documentos para la introducción y el soporte de la serie de normas ISO 9000: Orientación sobre el concepto y uso del enfoque basado en procesos para los sistemas de gestión (ISO/TC176/SC 2/\& 544R3). http://www.aenor.es/aenor/especial/iso9000

Unión Europea (2004). “Decisión 2004/387/CE del Parlamento Europeo y del Consejo, de 28 de abril de 2004, relativa a la prestación interoperable de servicios paneuropeos de administración electrónica al sector público, las empresas y los ciudadanos (Idabc)". Diario oficial, n. 181, 18 de mayo, pp. 25-35. http://eurlex.europa.eu/LexUriServ/LexUriServ.do?uri=OJ:L: 2004:181:0025:0035:ES:PDF

Unión Europea (2006). “Directiva 2006/123/CE del Parlamento Europeo y del Consejo de 12 de diciembre de 2006, relativa a los servicios en el mercado interior". Diario oficial, n. 376 de 12 de diciembre, pp. 36-68.

http://eur-lex.europa.eu/legal-content/ES/TXT/?uri=celex:32006L0123

Unión Europea (2009). “Decisión n. 922/2009/CE del Parlamento Europeo y del Consejo, de 16 de septiembre de 2009, relativa a las soluciones de interoperabilidad para administraciones europeas públicas (ISA)". Diario oficial, n. 260 de 3 de octubre, pp. 20-27.

http://eur-lex.europa.eu/legal-content/ES/TXT/?uri=CELEX\%3A32009D0922

European Commission (2011). European interoperability framework (EIF). Towards interoperability for European public services.

http://ec.europa.eu/isa/documents/eif_brochure_2011.pdf

Vida-Fernández, José (2010). “La administración electrónica en la Unión Europea”. Claves del gobierno local, n. 12, pp. 13-59. http://repositorio.gobiernolocal.es/xmlui/handle/10873/966

\section{Actualización del IF y del SJR}

En junio de 2016 SCImago y Thomson Reuters han publicado sendas actualizaciones de los indicadores de impacto de las revistas que indexan en sus respectivas bases de datos.

\section{SJR}

0.6

0.4

0.2

0 2007
$0.422(2015)$

El Impact Factor (IF) que elabora Thomson Reuters sobre las bases de datos Science Citation Index y Social Sciences Citation Index lambas integradas en la Web of Sciencel y que presenta en la base de datos Journal Citation Reports (JCR) también muestra un buen incremento de la popularidad de esta revista El profesional de la información: hemos pasado de

$$
\text { IF } 2014=0,356 \text { a IF } 2015=0,710(+99,4 \%)
$$

El grupo de investigación español SCImago, por encargo de Elsevier, elabora el indicador SCImago Journal Rank (SJR) basado en las citas que reciben las revistas indexadas en Scopus.

Esta revista El profesional de la información ha pasado de

$$
\text { SJR } 2014=0,374 \text { a SJR } 2015=0,422(+12,8 \%)
$$

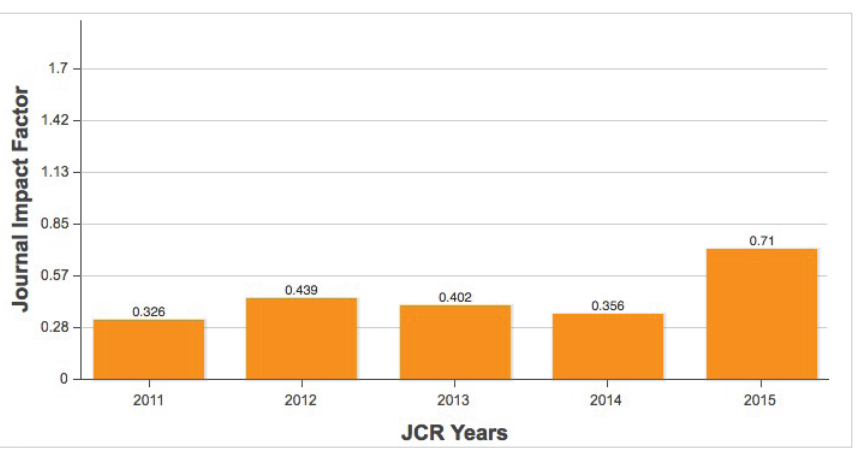

Damos las gracias a nuestros autores y a nuestros lectores, gracias a cuyo apoyo hemos podido hacer este avance en el ranking de revistas. 


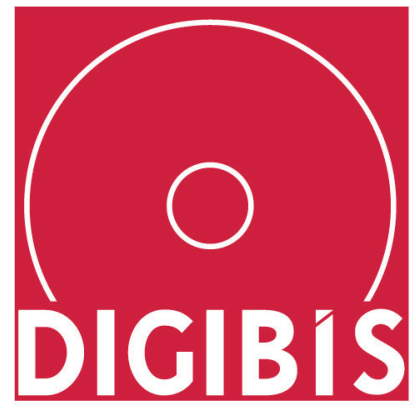

Digitalización enriquecida de fondo antiguo y patrimonial

Software de gestión para Bibliotecas, Archivos y Museos DIGIBIB ${ }^{\circ}$ DIGIARCH $^{\circ}$ DIGIMUS ${ }^{\circ}$

\section{Recolector OAI-PMH DIGIHUB'} de metadatos de diversos proveedores

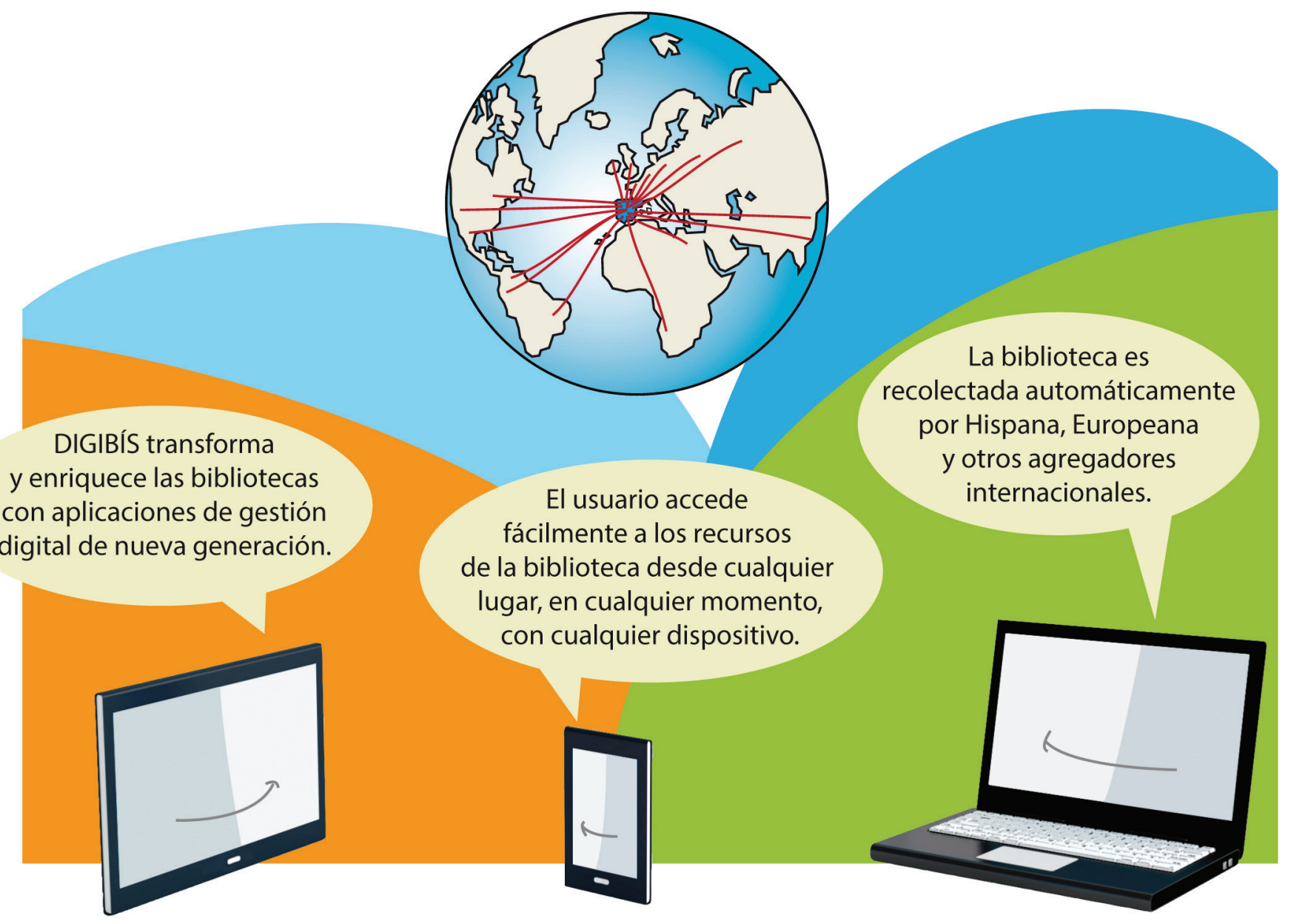

\section{¡Con estándares internacionales para un mundo enlazado y abierto!}

